\title{
MODULE AMENABILITY FOR SEMIGROUP ALGEBRAS
}

\author{
MASSOUD AMINI
}

\begin{abstract}
We extend the concept of amenability of a Banach algebra $A$ to the case that there is an extra $\mathfrak{A}$-module structure on $A$, and show that when $S$ is an inverse semigroup with subsemigroup $E$ of idempotents, then $A=\ell^{1}(S)$ as a Banach module over $\mathfrak{A}=\ell^{1}(E)$ is module amenable iff $S$ is amenable. When $S$ is a discrete group, $\ell^{1}(E)=\mathbb{C}$ and this is just the celebrated Johnson's theorem.
\end{abstract}

\section{INTRODUCTION}

The celeberated Johnson's theorem (in the discrete case) asserts that a discrete group $G$ is amenable if and only if the Banach algebra $\ell^{1}(G)$ is amenable. This fails to be true for discrete semigroups (even for the good cases like Clifford semigroups). Indeed Dunford and Namioka have shown that for a wide class of inverse semigroups (the class of $E$-unitary inverse semigroups) $\ell^{1}(S)$ is not amenable if the subsemigroup $E=E_{S}$ of idempotent elements is infinite [DN].

The concept of Johnson's amenability for Banach algebra has been a main stream in the theory of Banach algebras in the last fifty years. For some classes of operator algebras, however there have been some parallel concepts, among which one could mention the concept of central amenability for $C^{*}$-algebras [L2],[PR]. Also recently some research has been done on the relative amenability of Banach algebars [L1],[L3].

Here we develope the concept of module amenability for a class of Banach algebras which could somehow be considered as a generalization of all of the above approaches. In particular we apply this idea to the above mentioned problem and show that if $\ell^{1}(S)$ is considered appropriately as a $\ell^{1}(E)$-module, then its module amenability is equivalent to amenability of $S$, restoring the Johnson's theorem for the case of inverse semigroups.

The next section is devoted to the general theory of module amenability for Banach algebras in which we prove the analogues of the classical results on amenability of Banach algebras. Our main reference is $[\mathrm{P}]$, which in most cases we adapt almost the same proof. The details of proofs are given for the sake of completeness. In the last section, these results are used to prove the above mentioned version of the Johnson's theorem for inverse semigroups. We believe that this theory could well be applied to the case of topological (or measure) groupoids to get similar amenability results.

1991 Mathematics Subject Classification. Primary 43A07: Secondary 46H25.

Key words and phrases. amenability, module amenability, semigroup algebras.

Partially supported by a grant from University of Saskatchewan. 


\section{MODULE AMENABILITY}

Let $\mathfrak{A}$ be a Banach algebra and $A$ be a Banach $\mathfrak{A}$-module such that it has an associative product which makes it a Banach algebra which is compatible with the module action in the sense that

$$
\alpha .(a b)=(\alpha . a) b, \quad(\alpha \beta) . a=\alpha .(\beta . a) \quad(\alpha, \beta \in \mathfrak{A}, a, b \in A)
$$

and the same for the right action.

Definition 2.1. A is called module amenable (as an $\mathfrak{A}$-module, if ambiguity may happen) if for any Banach space $X$ which is at the same time a Banach A-module and a Banach $\mathfrak{A}$-module with the compatibility of actions

$$
(a . x) \cdot \alpha=a .(x . \alpha), \quad \alpha .(a . x)=(\alpha . a) \cdot x \quad(\alpha \in \mathfrak{A}, a \in A, x \in X)
$$

and the same for the other side actions, and each bounded map $D: A \rightarrow X^{*}$ with

$$
D(a+b)=D(a)+D(b), D(a b)=a \cdot D(b)+D(a) \cdot b \quad(a, b \in A),
$$

and

$$
D(\alpha . a)=\alpha . D(a), \quad D(a . \alpha)=D(a) . \alpha \quad(\alpha \in \mathfrak{A}, a \in A),
$$

there is an $x \in X^{*}$ such that $D(a)=a . x-x . a=: D_{x}(a) \quad(a \in A)$. Note that $D$ is not assumed to be $\mathbb{C}$-linear and so it is not necessarily an $\mathfrak{A}$-module homomorphism.

Note that $X^{*}$ is also a Banach module over $A$ and $\mathfrak{A}$ with compatible actions under the canonical actions of $A$ and $\mathfrak{A}$

$$
\alpha .(a . f)=(\alpha . a) \cdot f \quad\left(a \in A, f \in X^{*}, x \in X\right),
$$

and the same for the right actions. Here the canonical actions of $A$ and $\mathfrak{A}$ on $X^{*}$ are defined by

$$
(\alpha . f)(x)=f(x . \alpha), \quad(a . f)(x)=f(x . a) \quad\left(\alpha \in \mathfrak{A}, a \in A, f \in X^{*}, x \in X\right),
$$

and the same for right actions.

We call $A$-modules $X$ which have a compatible $\mathfrak{A}$-action as above, $A$ - $\mathfrak{A}$-modules, and derivations like $D$ in the above definition, the module derivations. Hence the above assertion is to say that if $X$ is an $A-\mathfrak{A}$-module, then so is $X^{*}$ under the canonical actions. Also we use the notation $Z_{\mathfrak{A}}\left(A, X^{*}\right)$ for the set of all module derivations $D: A \rightarrow X^{*}$, and $B_{\mathfrak{A}}\left(A, X^{*}\right)$ for those which are inner and $H_{\mathfrak{A}}^{1}\left(A, X^{*}\right)$ for the quotient group (which we call the first relative (to $\mathfrak{A}$ ) cohomology group of $\left.X^{*}\right)$. Hence $A$ is module amenable iff $H_{\mathfrak{A}}^{1}\left(A, X^{*}\right)=0$, for each $A$-A -module $X$.

Blanket Assumption: All over this paper we fix $A$ and $\mathfrak{A}$ as above, and use notations $X$ and $D$ for arbitrary module and derivation as in the above definition, unless they are otherwise specified explicitly.

Proposition 2.1. If $\mathfrak{A}$ has a bounded approximate identity, then amenability of $A$ implies its module amenability.

Proof By the Cohen's factorization theorem [DW] for $\mathfrak{A}$-modules $A$ and $X^{*}$, for each $a \in A, x \in X^{*}$ there are $\beta, \gamma \in \mathfrak{A}, b \in A$, and $y \in X^{*}$ such that $a=\beta . b$ and $x=\gamma \cdot y$. Therefore if $\left\{\alpha_{i}\right\}$ is a bounded approximate identity in $\mathfrak{A}$, then

$$
\begin{aligned}
D(\lambda a) & =D(\lambda(\beta . b))=\lim _{i} D\left(\lambda \alpha_{i} \cdot a\right)=\lim _{i} \lambda \alpha_{i} \cdot D(a)=\lim _{i} \lambda \alpha_{i} \cdot(\gamma \cdot y) \\
& =\lambda(\gamma \cdot y)=\lambda D(a)
\end{aligned}
$$

for each $\lambda \in \mathbb{C}, a \in A$. Hence $D$ is $\mathbb{C}$-linear, and so inner. 
As we will see later in section 3, the converse is false. Hence module amenability is somehow weaker than amenability. Indeed another example in section 3 shows that module amenability even does not imply the existence of a bounded approximate identity. However we have the following weaker notion which is implied by module amenability.

Definition 2.2. A bounded net $\left\{a_{i}\right\}$ in $A$ is called a bounded approximate commutator if

$$
\lim _{i}\left\|a_{i} a-a a_{i}\right\|=0 \quad(a \in A) .
$$

Clearly each bounded approximate identity is a bounded approximate commutator.

Proposition 2.2. If $A$ is module amenable then it has a bounded approximate commutator.

Proof Consider $X=A^{*}$. Then $X$ and $X^{*}$ are $A-\mathfrak{A}$-modules under the canonical actions

$$
(a . f)(b)=f(b a),(\alpha . f)(b)=f(b . \alpha) \quad\left(a, b \in A, \alpha \in \mathfrak{A}, f \in A^{*}\right),
$$

and

$$
(a . F)(f)=F(f . a),(\alpha . F)(f)=F(f . \alpha) \quad\left(a \in A, \alpha \in \mathfrak{A}, f \in A^{*}, F \in A^{* *}\right),
$$

with right actions defined similarly. Now consider the canonical embedding $\theta$ : $A \hookrightarrow A^{* *}$, then clearly $\theta \in Z_{\mathfrak{A}}\left(A, X^{*}\right)$, so there is $x \in A^{* *}$ such that $\theta(a)=$ $a . x-x . a \quad(a \in A)$. Take any norm bounded (by $C$ ) net $\left\{a_{i}\right\}$ in $A$ so that $\theta\left(a_{i}\right) \rightarrow x$ in $\sigma\left(A^{* *}, A^{*}\right)$, then it is easy to see that $a_{i} a-a a_{i} \rightarrow 0$ for each $a \in A$, in $\sigma\left(A, A^{*}\right)$. Hence given $\epsilon>0$ and finite subset $F \subseteq A$, there is a convex combination $a_{F, \epsilon}$ of elements of $F$, norm bounded by $C$, such that $\left\|a_{F, \epsilon} a-a a_{F, \epsilon}\right\|<\epsilon$, for each $a \in F$. Then $\left\{a_{F, \epsilon}\right\}_{(F, \epsilon)}$ forms a bounded approximate commutator for $A$.

Given $\mathfrak{A}$-modules $A$ and $B$, let $A \hat{\otimes}_{\mathfrak{A}} B$ be the projective module tensor product of $A$ and $B[\mathrm{R}]$. This is the quotient of the usual projective tensor product $A \hat{\otimes} A$ by the closed ideal $I$ generated by elements of the form $z . a \otimes b-a \otimes z . a$ for $z \in \mathfrak{A}, a, b \in A$. If $A$ and $B$ are also Banach algebras with compatible action, then so is $A \hat{\otimes}_{\mathfrak{A}} B$. Also $\left(A \hat{\otimes}_{\mathfrak{A}} B\right)^{*} \cong \mathfrak{L}_{\mathfrak{A}}\left(B, A^{*}\right)$, where the right hand side is the space of all $\mathfrak{A}$-module morphisms from $B$ to $A^{*}[\mathrm{R}]$. In particular $A \hat{\otimes}_{\mathfrak{A}} A$ is a Banach $A$ - $\mathfrak{A}$-module. Here the second $A$ in the tensor product is understood to be with with the opposite product. Consider $\omega \in \mathfrak{L}(A \hat{\otimes} A, A)$ defined by $\omega(a \otimes b)=$ $a b \quad(a, b \in A)$. Then both $\omega$ and its dual conjugate $\omega^{* *} \in \mathfrak{L}\left((A \hat{\otimes} A)^{* *}, A^{* *}\right)$ are Banach algebra homomorphisms. Now as $I$ is an ideal of $A \hat{\otimes} A$, so $\omega(I)$ is an ideal of $A$, and if $J$ is the closure of $\omega(I)$ we can define $\tilde{\omega}: A \hat{\otimes}_{\mathfrak{A}} A=A \hat{\otimes} A / I \rightarrow A / J$ by

$$
\tilde{\omega}(a \otimes b+I)=a b+J \quad(a, b \in A) .
$$

This extends to an element $\tilde{\omega} \in \mathfrak{L}\left(A \hat{\otimes}_{\mathfrak{A}} A, A / J\right)$ and both $\tilde{\omega}$ and its dual conjugate $\tilde{\omega}^{* *} \in \mathfrak{L}\left(\left(A \hat{\otimes}_{\mathfrak{A}} A\right)^{* *}, A^{* *} / J^{\perp \perp}\right)$ are $A$ - $\mathfrak{A}$-module homomorphisms.

Definition 2.3. $A$ bounded net $\left\{\tilde{u}_{i}\right\}$ in $A \hat{\otimes}_{\mathfrak{A}} A$ is called a module approximate diagonal if $\tilde{\omega}\left(\tilde{u}_{i}\right.$ is a (bounded) approximate identity of $A / J$ and $\lim _{i}\left\|\tilde{u}_{i} \cdot a-a . \tilde{u}_{i}\right\|=$ $0 \quad(a \in A)$. An element $\tilde{M} \in\left(A \hat{\otimes}_{\mathfrak{A}} A\right)^{* *}$ is called a module virtual diagonal if

$$
\tilde{\omega}^{* *}(\tilde{M}) \cdot a=a, \quad \tilde{M} \cdot a=a \cdot \tilde{M} \quad(a \in A) .
$$


Proposition 2.3. The following are equivalent:

(i) A has a module virtual diagonal

(ii) There is $M \in(A \hat{\otimes} A)^{* *}$ such that

$$
\omega^{* *}(M) . a-a \in J^{\perp \perp}, \quad M . a-a \cdot M \in I^{\perp \perp} \quad(a \in A),
$$

where $I$ is the closed ideal of $A \hat{\otimes} A$ generated by elements of the form $z . a \otimes b-a \otimes z . a$ for $z \in \mathfrak{A}, a, b \in A$, and $J=\omega \overline{(} I)$.

In particular if $A$ has a virtual diagonal, then it has a module virtual diagonal .

Proof If $M$ is as in $(i i)$, define $\tilde{M} \in\left(A \hat{\otimes}_{\mathfrak{A}} A\right)^{* *}=(A \hat{\otimes} A)^{* *} / I^{\perp \perp}$ by $\tilde{M}=$ $M+I^{\perp \perp}$. Then given $a \in A$, since $M . a-a \cdot M \in I^{\perp \perp}$, clearly $\tilde{M} \cdot a=a \cdot \tilde{M}$, and since $\omega^{* *}(M) \cdot a-a \in J^{\perp \perp}$ and $\tilde{\omega}^{* *}(\tilde{M})=\tilde{\omega}^{* *}\left(M+I^{\perp \perp}\right)=\omega^{* *}(M)+J^{\perp \perp}$, clearly $\tilde{\omega}^{* *}(\tilde{M}) \cdot a=a$.

Conversely, if $\tilde{M}$ is a module virtual diagonal, then choose any $M \in(A \hat{\otimes} A)^{* *}$ such that $\tilde{M}=M+I^{\perp \perp}$, then given $a \in A, M \cdot a-a \cdot M+I^{\perp \perp}=\tilde{M} \cdot a-a \cdot \tilde{M}=0 \in$ $(A \hat{\otimes} A)^{* *} / I^{\perp \perp}$, so $M . a-a \cdot M \in I^{\perp \perp}$. Also $\omega^{* *}(M) \cdot a-a+J^{\perp \perp}=\tilde{\omega}^{* *}(M) \cdot a-a=$ $0 \in A^{* *} / J^{\perp \perp}$, so $\omega^{* *}(M) . a-a \in J^{\perp \perp}$.

The following is proved similarly.

Proposition 2.4. The following are equivalent:

(i) A has a module approximate diagonal

(ii) There is a bounded net $\left\{u_{i}\right\} \in(A \hat{\otimes} A)$ such that for each $a \in A$ the nets $\left\{\omega\left(u_{i}\right) . a-a\right\}$ and $\left\{u_{i} . a-a . u_{i}\right\}$ converge to an element of $J$ and $I$, respectively.

In particular if $A$ has an approximate diagonal, then it has a module approximate diagonal .

Lemma 2.1. With above notations

(i) If $A$ is unital with unit $e$, then $H_{\mathfrak{A}}^{1}(A, X) \cong H_{\mathfrak{A}}^{1}(A, e X e)$,

(ii) $H_{\mathfrak{A}}^{1}(A \oplus \mathfrak{A}, X) \cong H_{\mathfrak{A}}^{1}(A, X)$,

(iii) If $A$ has bounded approximate identity, then $H_{\mathfrak{A}}^{1}\left(A, X^{*}\right) \cong H_{\mathfrak{A}}^{1}\left(A,(A X A)^{*}\right)$,

(iv) If $\mathfrak{A}$ has bounded approximate identity, then $H_{\mathfrak{A}}^{1}\left(A, X^{*}\right) \cong H_{\mathfrak{A}}^{1}\left(A,(\mathfrak{A} X \mathfrak{A})^{*}\right)$.

Proof $(i)$ Consider the $A-\mathfrak{A}$-module homomorphisms $i d, \ell, r: X \rightarrow X$ defined by $i d(x)=x, \ell(x)=e . x$ and $r(x)=x . e$. Then

$$
X \cong e X e \oplus(i d-r) \circ \ell(X) \oplus(i d-\ell) \circ r(X) \oplus(i d-r) \circ(i d-\ell)(X),
$$

with pairwise commuting projections. Now it is easy to check that each summand of the right hand side is a Banach $A-\mathfrak{A}$-module and that the first relative cohomology groups of the last three terms vanish.

(ii) Clearly $X$ is an $(A \oplus \mathfrak{A})-\mathfrak{A}$-module. Let $D$ be any module derivation from $A \oplus \mathfrak{A}$ to $X$, then under the identification $\mathfrak{A} \cong\{0\} \oplus \mathfrak{A}$, for each $a \in A$ and $\alpha, \beta \in \mathfrak{A}$ we have

$$
(a, \beta) \cdot D(0, \alpha)=D((a, \beta) \cdot(0, \alpha))-D((a, \beta)) \cdot \alpha=D((a, \beta) \cdot \alpha)-D((a, \beta)) \cdot \alpha=0 .
$$

Hence $D \circ \theta$ is a module derivation from $A$ to $X$, for the canonical embedding $\theta: A \rightarrow A \oplus \mathfrak{A}$. It is easy to see that $D \mapsto D \circ \theta$ lifts to an isomorphism of the corresponding relative cohomology groups.

(iii) Let $X_{1}=A X A$ and $X_{2}=A X$. As $A$ contains a bounded approximate identity $\left\{a_{i}\right\}$, Cohen's factorization theorem shows that $X_{1}, X_{2}$ are $A$ - $\mathfrak{A}$-modules. Now $A$ has a zero left action on $X_{2}^{\perp} \cong\left(X / X_{2}\right)^{*}$, so $H_{\mathfrak{A}}^{1}\left(A, X_{2}^{\perp}\right)=0$. Also $\left\{a_{i}\right\}$ is a 
bounded net in $\mathfrak{L}_{\mathfrak{A}}\left(X^{*}\right) \cong\left(X \hat{\otimes}_{\mathfrak{A}} X^{*}\right)^{*}$, so passing to a subnet we may assume that it is $w^{*}$-convergent to some $F \in \mathfrak{L}\left(X^{*}\right)$. Then

$$
<a . x, F(f)>=\lim _{i}<a . x, f . a_{i}>=\lim _{i}<a_{i} a . x, f>=<a . x, f>,
$$

for each $a \in A, f \in X^{*}, x \in X$. Hence $I-F$ is a projection of $X^{*}$ onto $X_{2}^{\perp}$, where $I$ is the identity map on $X^{*}$. Therefore $X^{*} \cong X_{2} \oplus X_{2}^{\perp}$ and so $H_{\mathfrak{A}}^{1}\left(A, X^{*}\right) \cong H_{\mathfrak{A}}^{1}\left(A, X_{2}^{*}\right)$. Now $H_{\mathfrak{A}}^{1}\left(A, X_{1}^{*}\right) \cong H_{\mathfrak{A}}^{1}\left(A, X_{2}^{*}\right)$ is similar.

(iv) Proof is quite similar to (iii).

Theorem 2.1. The following are equivalent

(i) $A$ is module amenable,

(ii) A has a module approximate diagonal,

(iii) A has a module virtual diagonal.

Proof The equivalence of $(i i)$ and (iii) follows exactly like in the classical case [P, Lemma 1.6]. Assume that $A$ has a module virtual diagonal $\tilde{M} \in\left(A \hat{\otimes}_{\mathfrak{A}} A\right)^{* *}$ and let $M \in(A \hat{\otimes} A)^{* *}$ be the corresponding element as in (ii) of Proposition 2.3. We may assume that $\tilde{M}$ is a $w^{*}$-limit point of a module approximate diagonal $\left\{\tilde{u}_{i}\right\}$. Take any Banach $A$ - $\mathfrak{A}$-module $X$ and any module derivation $D: A \rightarrow X^{*}$. By above lemma, we may assume that $X$ is essential, that is $X=A X A$. To each $x \in X$, there corresponds $F_{x} \in(A \hat{\otimes} A)^{*}$ via

$$
F_{x}(a \otimes b)=<x, a \cdot D(b)>\quad(a, b \in A) .
$$

For each $a \in A, x \in X$. Then $D(a) . x$ could thought as an element of $A^{*}$ via

$$
<D(a) \cdot x, b>=<D(a), b \cdot x>\quad(b \in A),
$$

and it is easy to see that

$$
F_{(a \cdot x-x \cdot a)}=a \cdot F_{x}-F_{x} \cdot a+\omega^{*}(D(a) \cdot x) \quad(a \in A, x \in X),
$$

(see proof of $[\mathrm{P}, 1.7])$. Putting $\tilde{F}_{x}=F_{x}+I^{\perp}$ and $f(x)=<F_{x}, M>\quad(x \in X)$, we have $\tilde{F}_{x} \in\left(A \hat{\otimes}_{\mathfrak{A}} A\right)^{*}$ and $f \in X^{*}$, it is easy to check that

$$
\tilde{F}_{(a . x-x . a)}=a \cdot \tilde{F}_{x}-\tilde{F}_{x} \cdot a+\tilde{\omega}^{*}\left(D(a) \cdot x+J^{\perp}\right) \quad(a \in A, x \in X),
$$

and so

$$
\begin{aligned}
<x, D_{f}(a)> & =<\tilde{F}_{(a \cdot x-x \cdot a)}, \tilde{M}> \\
& =<a \cdot \tilde{F}_{x}-\tilde{F}_{x} \cdot a, \tilde{M}>+<\tilde{\omega}^{*}\left(D(a) \cdot x+J^{\perp}\right), \tilde{M}> \\
& =<\tilde{F}_{x}, \tilde{M} \cdot a-a \cdot \tilde{M}>+\lim _{i}<x \cdot \tilde{\omega}\left(\tilde{u}_{i}\right), D(a)>=<x, D(a)>,
\end{aligned}
$$

where the last equality is because $X$ is essential. Hence $D=D_{f}$.

Conversely assume that $A$ is module amenable; then by Proposition 2.2 , it has a bounded approximate identity $\left\{a_{i}\right\}$. Put $\dot{a}_{i}=a_{i}+J \in A / J$, then passing to a subnet, we may assume that $\left\{\dot{a}_{i} \otimes \dot{a}_{i}\right\}$ is $w^{*}$-convergent to an element $N \in$ $\left(A \hat{\otimes}_{\mathfrak{A}} A\right)^{* *}$. Clearly $\tilde{\omega}$ vanishes on the range of $D_{N}$ and so $D_{N}$ could be regarded as a module derivation of $A$ into the $A$ - $\mathfrak{A}$-module $K=\operatorname{ker}\left(\tilde{\omega}^{* *}\right)$. By module amenability of $A$, there is $N^{\prime} \in K$ such that $D_{N}=D_{N^{\prime}}$, so $M=N-N^{\prime}$ is a module virtual diagonal .

Proposition 2.5. If $A$ and $B$ are Banach algebras and Banach $\mathfrak{A}$-modules with compatible actions, and there is a continuous Banach algebra homomorphism and 
module morphism $\varphi$ from $A$ onto a dense subset of $B$, and $A$ is module amenable, then so is $B$.

Proof If $X$ is any $B$-A -module then via $\varphi, X$ could be regarded as an $A$ $\mathfrak{A}$-module, and each module derivations $D: B \rightarrow X^{*}$, gives a module deivation $D \circ \varphi: A \rightarrow X^{*}$, which is inner. By density of range of $\varphi$ and continuity of $D$, then $D$ would be inner.

Next assume that $A$ has a bounded approximate identity $a_{i}$ and consider the algebra of $\mathfrak{A}$-multipliers of $A$

$M_{\mathfrak{A}}(A)=\left\{\left(T_{1}, T_{2}\right): T_{1}, T_{2} \in \mathfrak{L}_{\mathfrak{A}}(A): T_{1}(a b)=T_{1}(a) b, T_{2}(a b)=a T_{2}(b)(a, b \in A)\right\}$.

Then $M_{\mathfrak{A}}(A)$ is an $A$-A $\mathfrak{A}$-module and $A$ embeds in $M_{\mathfrak{A}}(A)$ via $a \mapsto\left(S_{a}, T_{a}\right)$, where $S_{a}(b)=a b, T_{a}(b)=b a \quad(a, b \in A)$. For any element $T=\left(T_{1}, T_{2}\right)$ of $M_{\mathfrak{A}}(A)$ it is easy to see that $\left\|T_{1}\right\|=\left\|T_{2}\right\|$ and if we put $\|T\|$ equal to this common value, then $M_{\mathfrak{A}}(A)$ becomes a Banach $A$-A-module. Also for each $A$-A-module $X, M_{\mathfrak{A}}(A)$ acts on $X$ via

$$
T . x=\lim _{i} T_{1}\left(a_{i}\right) \cdot x, \quad x \cdot T=\lim _{i} x \cdot T_{2}\left(a_{i}\right) \quad\left(x \in X, T=\left(T_{1}, T_{2}\right) \in M_{\mathfrak{A}}(A)\right),
$$

which makes $X$ a $M_{\mathfrak{A}}(A)$ - $\mathfrak{A}$-module. Also given a module derivation $D: M_{\mathfrak{A}}(A) \rightarrow$ $X^{*}$, the restriction $D^{\prime}$ of $D$ to $A$ is a module derivation on $A$.

Proposition 2.6. With the above notation, if $A$ has a bounded approximate identity, then $H_{\mathfrak{A}}^{1}\left(M_{\mathfrak{A}}(A), X^{*}\right) \cong H_{\mathfrak{A}}^{1}\left(A, X^{*}\right)$.

Proof It is enough to show that if the right hand side is $\{0\}$, then so is the left hand side. If $D$ and $D^{\prime}$ are as above, then $D^{\prime}=D_{x}$, for some $x \in X^{*}$. Put $D_{i}=T_{1}\left(a_{i}\right) \cdot x-x \cdot T_{2}\left(a_{i}\right)$, for each index $i$ and $T=\left(T_{1}, T_{2}\right) \in M_{\mathfrak{A}}(A)$, then the net $D_{i}$ is uniformely norm bounded, and so, passing to a subnet, we may assume that it converges to some $D_{0} \in \mathfrak{L}_{\mathfrak{A}}\left(M_{\mathfrak{A}}(A), X^{*}\right)$ in $\sigma\left(X^{*}, M_{\mathfrak{A}}(A)\right)$. Then it is easy to show that $D_{0}=D$ and since clearly $D_{0}=D_{x}$ on $A$, so by the continuity of these derivations in the strict topology of $M_{\mathfrak{A}}(A)$ and density of $A$ in $M_{\mathfrak{A}}(A)$ in the strict topology, we get $D=D_{x}$ on $M_{\mathfrak{A}}(A)$.

Corollary 2.1. Let $J$ be a closed ideal of $A$ which is $\mathfrak{A}$-invariant, i.e. $\mathfrak{A} . J \subseteq J$. If $J$ has a bounded approximate identity and $A$ is module amenable then $J$ is also module amenable.

Proof Let $X$ be an essential Banach $J$ - $\mathfrak{A}$-module and $D: M_{\mathfrak{A}}(J) \rightarrow X^{*}$ be a bounded module derivative. By definition of $M_{\mathfrak{A}}(J)$, there is an $\mathfrak{A}$-module morphism $\varphi: A \rightarrow M_{\mathfrak{A}}(J)$ and $D \circ \varphi$ is a module derivation on $A$, so it is inner. Hence $D$ is inner on $J$. By the same argument as above $D$ is inner on $M_{\mathfrak{A}}(J)$ and so the result follows from the above proposition.

Corollary 2.2. If $J$ is an $\mathfrak{A}$-invariant closed ideal of $A$ with bounded approximate identity, then $A$ is module amenable iff both $J$ and $A / J$ are module amenable.

Proof One direction is trivial, for the other, assume that both $J$ and $A / J$ are module amenable and let $D: A \rightarrow X^{*}$ be any bounded module derivative, then the restriction $D^{\prime}$ of $D$ to $J$ is inner. It is easy to see that the range of $D-D^{\prime}$ vanishes on both $J X$ and $X J$. Now The close submodule $X_{J}$ of $X$ generated by $J X \cup X J$ is a Banach $A$-A-module and $D-D^{\prime}: A \rightarrow \hat{X}_{J} \cong\left(X / X_{J}\right)^{*}$ is a module derivative which vanishes on $J$, so it could be regarded as a module deivative on $A / J$, and so it is inner. Hence $D$ is inner. 
Definition 2.4. Given a net of Banach algebras and $\mathfrak{A}$-modules $\left\{A_{i}\right\}$ with compatible actions, we say that they are simultaneously module amenable if there is a constant $c>0$ such that for each index $i$, each $A_{i}$-A-module $X_{i}$, each module derivation $D_{i}: A_{i} \rightarrow X_{i}^{*}$, there is $x_{i} \in X_{i}^{*}$ such that $D_{i}=D_{x_{i}}$ and $\left\|x_{i}\right\| \leq c\left\|D_{i}\right\|$.

Proposition 2.7. If a direct system $\left\{A_{i}\right\}$ is simulteneously module amenable, then $A:=\varliminf_{\longrightarrow} A_{i}$ is module amenable .

Proof We may assume that $A_{i} \subseteq A_{i} \subseteq A \quad(i \leq i)$ and $A=\left(\cup_{i} A_{i}\right)^{-}$. Let $X$ be a Banach $A-\mathfrak{A}$-module and $D: A \rightarrow X^{*}$ is a bounded module derivative, then the restriction $D_{i}$ of $D$ to $A_{i}$ is a module derivative on $A_{i}$, so there is $c>0$ and $z_{i} \in X^{*}$ such that $D=D_{z_{i}}$ on $A_{i}$ and $\left\|z_{i}\right\| \leq c\|D\|$. Passing to a subnet we may assume that $\left\{z_{i}\right\}$ is $w^{*}$-convergent to some $z \in X^{*}$. Take any $a \in \cup_{i} A_{i}$, then $a \in A_{i} \quad\left(i>i_{0}\right)$, for some index $i_{0}$. Then

$$
\begin{aligned}
<D_{z}(a), x> & =<z, x . a-a . x>=\lim _{i}<x . a-a . x, z_{i}> \\
& =\lim _{i}<D_{z_{i}}(a), x>=<D(a), x>\quad\left(a \in A, x \in X^{*}\right),
\end{aligned}
$$

so $D=D_{z}$ on a dense subset of $A$, and so on $A$.

Next we consider the module amenability of the module tensor product.

Proposition 2.8. If $A$ and $B$ are module amenable then so is $A \hat{\otimes}_{\mathfrak{A}} B$.

proof $A s A$ and $B$ admit bounded approximate identity by Proposition 2.2, so does $A \hat{\otimes}_{\mathfrak{A}} B$. Take any essential Banach $A \hat{\otimes}_{\mathfrak{A}} B-\mathfrak{A}$-module $X$. The mappings $a \mapsto \sigma_{a}$ and $b \mapsto \tau_{b}$ defined by

$$
\sigma_{a}(c \otimes d)=a c \otimes d, \quad \tau_{b}(c \otimes d)=c \otimes b d \quad(a, c \in A, b, d \in B),
$$

extend and then lift to commuting continuous Banach algebra homomorphisms and $\mathfrak{A}$-module morphisms of $A$ and $B$ onto closed subalgebras $A_{1}$ and $B_{1}$ of $M_{\mathfrak{A}}\left(A \hat{\otimes}_{\mathfrak{A}} B\right)$, and $X$ is then a Banach $A_{1}-\mathfrak{A}$-module and $B_{1}-\mathfrak{A}$-module. Now each module derivative $D: A \hat{\otimes}_{\mathfrak{A}} B \rightarrow X^{*}$ gives rise to some module derivative $D^{\prime}$ : $M_{\mathfrak{A}}\left(A \hat{\otimes}_{\mathfrak{A}} B\right) \rightarrow X^{*}$, whose restriction $D_{1}$ to $B_{1}$ is inner, say $D^{\prime}=D_{x}$ on $B_{1}$, for some $x \in X^{*}$. Consider $d: X \rightarrow \mathfrak{L}_{\mathfrak{A}}\left(B_{1}, X\right)$, which sends each $y \in X$ to the restriction of $D_{y}$ to $B_{1}$. Then the fact that $D^{\prime}-D_{x}$ is zero on $B_{1}$ implies that $D^{\prime}-D_{x}$ is sending $A_{1}$ into $\operatorname{Im}(d)^{\perp} \cong(X / \operatorname{Im}(d))^{*}$ (since $\sigma$ and $\tau$ commute). Now $\operatorname{Im}(d)$ and so $Y:=X / \operatorname{Im}(d)$ is an $A_{1}-\mathfrak{A}$-module (again since $\sigma$ and $\tau$ commute), and so $D^{\prime}-D_{x}=D_{y}$ on $A_{1}$, for some $y \in Y$. But both sides of the last equality are equal zero on $B_{1}$, and so by the fact that $A_{1} \cup B_{1}$ is dense in $M_{\mathfrak{A}}\left(A \hat{\otimes}_{\mathfrak{A}} B\right), D^{\prime}$ is inner on $M_{\mathfrak{A}}\left(A \hat{\otimes}_{\mathfrak{A}} B\right)$, so $D$ is inner on $A \hat{\otimes}_{\mathfrak{A}} B$.

Proposition 2.9. If $\mathfrak{A}$ is unital and $B$ is a unital amenable Banach algebra, then $A:=B \hat{\otimes}_{\mathfrak{A}} \mathfrak{A}$ is module amenable. In particular, if $\mathfrak{A}$ is unital then it is module amenable as a module over itself.

Proof As $\mathfrak{A}$ and $B$ are unital, $B$ and $\mathfrak{A}$ could be identified with a subalgebra of $A$. Given $A$ - $\mathfrak{A}$-module $X$, and bounded module derivative $D: A \rightarrow X^{*}, X$ is also a $B$ - $\mathfrak{A}$-module and the restriction $D_{1}$ of $D$ to $B$ is a module derivative. As $B$ is unital, $D$ is a derivation, and so inner. Also clearly the restriction $D_{2}$ of $D$ to $\mathfrak{A}$ is 
the zero map (as $\mathfrak{A}$ is unital). Now for each $b \in B, \alpha \in \mathfrak{A}$,

$$
\begin{aligned}
D(b \otimes \alpha) & =(1 \otimes \alpha) D_{1}(b)+D_{2}(\alpha) \cdot b=(1 \otimes \alpha)(b . x-x \cdot b)+0 \\
& =(b \otimes \alpha) \cdot x-x \cdot(b \otimes \alpha)=D_{x}(b \otimes \alpha) .
\end{aligned}
$$

Now as $\mathfrak{A}$ is unital, $D$ is indeed linear, hence $D=D_{x}$ on $B \hat{\otimes} \mathfrak{A}$.

Using this result we can now provide a family of examples of module amenable Banach algebras, Here are some:

Example 2.1. (i) If $\Omega$ is a compact topological space, then $C(\Omega, \mathfrak{A}) \cong C(\Omega) \hat{\otimes} \mathfrak{A}$ is module amenable.

(ii) If $\mathfrak{H}$ is a separable Hilbert space, then $(\mathfrak{K}(\mathfrak{H}) \oplus \mathbb{C} I) \hat{\otimes} \mathfrak{A} \cong(\mathfrak{K}(\mathfrak{H}) \hat{\otimes} \mathfrak{A}) \oplus \mathfrak{A}$ is module amenable.

(iii) If $G$ is a discrete amenable group, then $\ell^{1}(G) \hat{\otimes} \mathfrak{A}$ is module amenable, in particular, for $\mathfrak{A}=\ell^{1}(H)$, where $H$ is a subgroup of $G, \ell^{1}(G \times H)$ is module amenable as an $\ell^{1}(H)$-module.

\section{MOdUlE AMENABiLity OF SEMigRoup ALGEBRAS}

In this section we consider an important example which was the motivation for writing this paper. We consider an inverse semigroup $S$ with idempotents $E$ and show that $\ell^{1}(S)$ is module amenable as an $\ell^{1}(E)$-module if and only if $S$ is amenable.

Definition 3.1. A discrete semigroup $S$ is called an inverse semigroup if for each $x \in S$ there is a unique element $x^{*} \in S$ such that $x x^{*} x=x$ and $x^{*} x x^{*}=x^{*}$. An element $e \in S$ is called an idempotent if $e=e^{*}=e^{2}$. The set of idempotent elements of $S$ is denoted by $E$.

Definition 3.2. A discrete semigroup $S$ is called amenable if there is an invariant mean on $\ell^{\infty}(S)$, namely an element $m \in \ell^{\infty}(S)^{*}$ such that $m(1)=\|m\|=1$ and $m(s . f)=m(f . s)=m(f) \quad\left(s \in S, f \in \ell^{\infty}(S)\right)$, where

$$
f . s(t)=f(t s), \quad s . f(t)=f(s t) \quad\left(s, t \in S, f \in \ell^{\infty}(S)\right) .
$$

It is easy to see that $E$ is indeed a commutative subsemigroup of $S$. In particular $\ell^{1}(E)$ could be regarded as a subalgebra of $\ell^{1}(S)$, and thereby $\ell^{1}(S)$ is a Banach algebra and a Banach $\ell^{1}(E)$ module with compatible action. The same then would be true for $\ell^{\infty}(S)$. Of course one may change the action of $\ell^{1}(E)$ on $\ell^{1}(S)$ to get different module amenability results. One lesson we learned from the proof of Proposition 2.2 was that sometimes it is helpful to consider the action from one side to be some sort of trivial action (zero action in that case). We adapt that idea here and let $\ell^{1}(E)$ act from right on $\ell^{1}(S)$ by multiplication and as identity from left, that is

$$
\delta_{e} . \delta_{s}=\delta_{s}, \delta_{s} . \delta_{e}=\delta_{s} e=\delta_{s} * \delta_{e} \quad(s \in S, e \in E) .
$$

Lemma 3.1. With the above notation

(i) $\ell^{1}(S) \hat{\otimes}_{\ell^{1}(E)} \ell^{1}(S) \cong \ell^{1}(S \times S) / I$, where $I$ is the closed ideal of $\ell^{1}(S \times S)$ which is closed linear span of the set of elements of the form $\delta_{(s e t, x)}-\delta_{(s t, x)}$, where $s, t, x \in S$ and $e \in E$.

$(i i)\left(\ell^{1}(S) \hat{\otimes}_{\ell^{1}(E)} \ell^{1}(S)\right)^{*} \cong \ell^{\infty}(S \times S) / I^{\perp}$, where

$$
I^{\perp}=\left\{f \in \ell^{\infty}(S \times S): f(\text { set }, x)=f(s t, x) \quad(s, t, x \in S, e \in E)\right\} .
$$


Proof $(i)$ follows directly from the definition of the module projective tensor product. For $(i i)$, if $f \in I^{\perp}$, then for each $s, t, x \in S$ and $e \in E$,

$$
\begin{aligned}
0 & =<f, \delta_{(s e t, x)}-\delta_{(s t, x)}>=\sum_{u, v} f(u, v)\left(\delta_{(s e t, x)}(u, v)-\delta_{(s t, x)}(u, v)\right) \\
& =f(s e t, x)-f(s t, x) .
\end{aligned}
$$

Conversely, if $f$ satisfies the given relation, then clearly $\langle f, u\rangle=0$ for each $u \in I$ which is a finite linear combination of elements of the form $\delta_{(s e t, x)}-\delta_{(s t, x)}$, where $s, t, x \in S$ and $e \in E$. By continuity, then $f \in I^{\perp}$.

Consider $\omega: \ell^{1}(S) \hat{\otimes} \ell^{1}(S)=\ell^{1}(S \times S) \rightarrow \ell^{1}(S)$ defined by

$$
\omega(g)(s, t)=g(s t) \quad\left(s, t \in S, g \in \ell^{1}(S)\right),
$$

then

Now if

$$
\omega^{*}(h)(s, t)=h(s t) \quad\left(s, t \in S, h \in \ell^{\infty}(S)\right)
$$

$$
f . s\left(t, t^{\prime}\right)=f\left(s t, t^{\prime}\right) \quad s . f\left(t, t^{\prime}\right)=f\left(t, t^{\prime} s\right) \quad\left(s, t, t^{\prime} \in S, f \in \ell^{\infty}(S \times S)\right),
$$

and for $M \in \ell^{\infty}(S \times S)^{* *}$ and $f \in \ell^{\infty}(S \times S)$,

$$
M . s(f)=M(s . f), \quad s . M(f)=M(f . s),
$$

then $M$ is a virtual diagonal of $\ell^{1}(S)$ if and only if, for each $s \in S$

$$
M . s=s . M, \quad \omega^{* *}(M) . s=s,
$$

on $\ell^{\infty}(S \times S)$, where in the second equality the left hand side is defined by

$$
\omega^{* *}(M) . s(h)=M\left(s .\left(\omega^{*}(h)\right)\right) \quad\left(s \in S, h \in \ell^{\infty}(S)\right),
$$

and the right hand side is the functional of evaluation at $s$ on $\left.\ell^{\infty}(S)\right)$ [DN]. Now if $I$ is as in the above lemma and $J=\omega(I)^{-}$, then by Proposition 2.3, $M \in \ell^{1}(S \times S)^{* *}=$ $\ell^{\infty}(S \times S)^{*}$ gives rise to a module virtual diagonal for $\ell^{1}(S)$ if and only if, for each $s \in S$ the equalities

$$
M . s=s . M, \quad \omega^{* *}(M) . s=s,
$$

hold on $I^{\perp} \subseteq \ell^{\infty}(S \times S)$ and $J^{\perp}=\omega(I)^{\perp} \subseteq \ell^{\infty}(S)$, respectively, where

$$
J^{\perp}=\omega(I)^{\perp}=\left\{h \in \ell^{\infty}(S): h(\text { set })=h(s t) \quad(s, t \in S, e \in E)\right\} .
$$

Such an element $M$ exists if and only if $\ell^{1}(S)$ is module amenable.

Next consider the congruence relation $\sim$ on $S$ defined by $s \backsim t$ if and only if there is $e \in E$ such that $s e=t e$. The quotient semigroup $G_{S}:=S / \backsim$ is then a group. It is indeed the maximal group homomorphic image of $S[\mathrm{Mu}]$. Also the inverse semigroup $S$ is amenable if and only if the discrete group $G_{S}$ is amenable $[\mathrm{DN}]$.

Lemma 3.2. With above notation, $\ell^{1}\left(G_{S}\right)$ is a quotient of $\ell^{1}(S)$ and so the above action of $\ell^{1}(E)$ on $\ell^{1}(S)$ lifts to an action of $\ell^{1}(E)$ on $\ell^{1}\left(G_{S}\right)$, making it a Banach $\ell^{1}(E)$-module.

Proof Consider the quotient map $\pi: S \rightarrow G_{S}$, then extending $\pi$ by linearity and noting that for each $n \geq 1$, each $c_{1}, \ldots, c_{n} \in \mathbb{C}$, and each $s_{1}, \ldots, s_{n} \in S$

$$
\left\|\sum_{i=1}^{n} c_{i} \delta_{\pi\left(s_{i}\right)}\right\|_{1}=\left\|\sum_{i=1}^{n} c_{i} \delta_{s_{i}}\right\|_{1}
$$


one can extend $\pi$ to a continuous Banach algebra epimomorphism $\pi: \ell^{1}(S) \rightarrow$ $\ell^{1}\left(G_{S}\right)$.

Lemma 3.3. With the setup of above lemma, $\ell^{1}\left(G_{S}\right)$ is module amenable if and only if it is amenable.

Proof The left action of $\ell^{1}(E)$ on $\ell^{1}(S)$ and so on $\ell^{1}\left(G_{S}\right)$ is trivial. As for the right action, we have

$$
\delta_{\pi(s)} \cdot \delta_{e}=\delta_{\pi(s e)}=\delta_{\pi(s)} \quad(s \in S, e \in E)
$$

Hence the right action is also trivial and so

$$
\ell^{1}\left(G_{S}\right) \hat{\otimes}_{\ell^{1}(E)} \ell^{1}\left(G_{S}\right) \cong \ell^{1}\left(G_{S}\right) \hat{\otimes} \ell^{1}\left(G_{S}\right)
$$

and the result follows from Proposition 2.3.

Now we are ready to prove the main result of this section.

Theorem 3.1. Let $S$ be an inverse semigroup with idempotents $E$. Consider $\ell^{1}(S)$ as a Banach module over $\ell^{1}(E)$ with the multiplication right action and the trivial left action. Then $\ell^{1}(S)$ is module amenable if and only if $S$ is amenable.

proof If $\ell^{1}(S)$ is module amenable then so is $\ell^{1}\left(G_{S}\right)$ by Lemma 3.2 and Proposition 2.5 . Hence $\ell^{1}\left(G_{S}\right)$ is amenable by above Lemma, and so $G_{S}$ is amenable by Johnson's theorem. Therefore $S$ is amenable by [DN, thm.1]. by

Conversely if $\mu$ is a right invariant mean on $S$ and $M$ is defined on $\ell^{\infty}(S \times S)$

$$
M(f)=\int_{S} f\left(s^{*}, s\right) d \mu(s),
$$

then $M$ is clearly a bounded linear functional and $M(1 \otimes 1)=\mu(1)=1$. Also for each $s \in S$ and $f \in \ell^{\infty}(S \times S)$,

$$
\begin{aligned}
s . M(f)=M(f . s) & =\int_{S} f\left(s t^{*}, t\right) d \mu(t) \\
& =\int_{S} f\left(s(t s)^{*}, t s\right) d \mu(t) \\
& =\int_{S} f\left(s s^{*} t^{*}, t s\right) d \mu(t) \\
& =\int_{S} f\left(\left(t s s^{*}\right)^{*},\left(t s s^{*}\right) s\right) d \mu(t) \\
& =\int_{S} f\left(t^{*}, t s\right) d \mu(t) \\
& =M(s . f)=M . s(f),
\end{aligned}
$$

and for each $s \in S$ and $f \in J \subseteq \ell^{\infty}(S \times S)$, 


$$
\begin{aligned}
\omega^{* *}(M) . s(f) & =\omega^{* *} M(f . s) \\
& =M\left(\omega^{*}(f . s)\right)=\int_{S} \omega^{*}(f . s)\left(t^{*}, t\right) d \mu(t) \\
& =\int_{S} f . s\left(t^{*} t\right) d \mu(t)=\int_{S} f\left(s t^{*} t\right) d \mu(t) \\
& =f(s) \int_{S} d \mu(t)=f(s),
\end{aligned}
$$

where the last equality between integrals is because by the discussion after Lemma $3.1, f(s e)=f(s)$, for each $e \in E$. Hence $M$ gives rise to a module virtual diagonal for $\ell^{1}(S)$ and so $\ell^{1}(S)$ is module amenable .

Now we are ready to give the two countraexamples mentioned in section 2. For the second example we use the fact that if $S$ satisfies the $D_{k}$ condition of Duncan and Namioka, for some positive integer $k$ if and only if $\ell^{1}(E)$ has a bounded approximate identity if and only if $\ell^{1}(S)$ has a bounded approximate identity[DN].

Example 3.1. $(i)$ There is an inverse semigroup $S$ for which $\ell^{1}(S)$ is module amenable but not amenable .

(ii) There is an inverse semigroup $S$ for which $\ell^{1}(S)$ is module amenable but has no bounded approximate identity .

Proof For the first example, take any amenable inverse semigroup $S$ with infinite number of idempotents (there are a lot of them among Clifford semigroups). Then $\ell^{1}(S)$ is module amenable by above theorem, but not amenable [DN]. For the second example take any Brandt semigroup $S$ of an amenable group over an infinite index set then clearly $S$ is amenable and so again $\ell^{1}(S)$ is module amenable by above theorem, but it has no bounded approximate identity [DN].

Next we can consider the module amenability of the reduced semigroup $C^{*}$ algebra $C_{r}^{*}(S)$ and the semigroup von Neumann algebra $\mathrm{VN}(\mathrm{S})$ generated by $C_{r}^{*}(S)$. We refer the reader to $[\mathrm{Pa}]$ for definitions. Here we only need to know that $C_{r}^{*}(S)$ is a homomorphic image of $\ell^{1}(S)[\mathrm{Pa}]$. Therefore we may consider $C_{r}^{*}(S)$ as an $\ell^{1}(E)$-module (with the induced actions from the action of $\ell^{1}(E)$ on $\ell^{1}(S)$ as in the above theorem). Also $\mathrm{VN}(\mathrm{S})$ is homomorphic image of the second conjugate of $C_{r}^{*}(S)$ and so carries induced action of $\ell^{1}(E)$ similarly. Now in this setting we have the following two partial results.

Corollary 3.1. With the setting of the above theorem, if $S$ is amenable then $C_{r}^{*}(S)$ is module amenable.

Proof If If $S$ is amenable, then $C_{r}^{*}(S)$ is module amenable by above theorem and Proposition 2.3.

Corollary 3.2. With the setting of the above theorem, if every maximal subgroup of $S$ is amenable and $S$ satisfies the $D_{k}$ condition of Duncan and Namioka, for some positive integer $k$, then $V N(S)$ is module amenable.

Proof Under the first condition $V N(S)$ is amenable $[\mathrm{Pa}]$ and under the second condition $\ell^{1}(E)$ has a bounded approximate identity [DN]. Hence the result follows from Proposition 2.1. 
Acknowledgement: This paper is prepared while the author was visiting the department of Mathematics and Statistics of University of Saskatchewan. I would like to thank the hospitality I have received there. In particular I would like to thank Dr. M. Khoshkam and Dr. David Cowan for many stimulating discussions.

\section{REFERENCES}

[DN] J. Duncan, I. Namioka, Amenability of Inverse semigroups and their semigroup algebras. Proceedings of the Royal Society of edinburgh, 80A (1978) 309-321.

[L1] Z.A. Lykova, Structure of of Banach algebras with trivial central cohomology. J. Operator Theory 28 (1992) 147-165.

[L2] Z.A. Lykova, Ordinary and central amenability of $C^{*}$-algebras. Russian Math. Surveys 48 (1993) 1, 175-177.

[L3] Z.A. Lykova, Relative cohomology of Banach algebras. J. Operator Theory 41 (1999) 23-53.

[M] W.D. Munn, A class of irreducible matrix representations of an arbitrary inverse semigroup. Proc. Glasgow Math. Assoc. 5 (1961) 41-48.

[P] J.P Pier, Amenable Banach algebras, Pitman research notes in mathematical series 172, Longeman, Essex, 1988.

[Pa] A.L.T. Paterson, Groupoids, inverse semigroups, and their operator algebras, Birkhauser, Boston, 1999.

[PR] J. Phillips, I. Raeburn, Central cohomology of $C^{*}$-algebras. J. London Math. Soc. (2) 28 (1983) 365-375.

[R] M. A. Rieffel, Induced Banach representations of Banach algebras and locally compact groups. J. Func. Analysis 1 (1967) 443-491.

[DW] R.S. Doran, J. Wichmann, Approximate identities and factorization in Banach modules. Lecture Notes in Mathematics 768, Springer, berlin, 1979.

Department of Mathematics and Statistics, University of Saskatchewan, 106 Wiggins Road, Saskatoon, Saskatchewan, Canada S7N 5E6, mamini@math.usask.ca 